MATHEMATICS OF COMPUTATION

Volume 74 , Number 250 , Pages $953-965$

S 0025-5718(04)01671-0

Article electronically published on May 24, 2004

\title{
DETERMINANT FORMULAS FOR CLASS NUMBERS IN FUNCTION FIELDS
}

\author{
HWANYUP JUNG, SUNGHAN BAE, AND JAEHYUN AHN
}

\begin{abstract}
In this paper, by extending Kucera's idea to the function field case, we obtain several determinant formulas involving the real class number and the relative class number of any subfield of cyclotomic function fields. We also provide several examples using these determinant formulas.
\end{abstract}

\section{INTRODUCTION}

In the classical case, there are a lot of determinant formulas (Maillet's determinant, Demjanenko matrix) involving the relative class number for an imaginary abelian number field. For these determinant formulas, we refer to Kučera's paper [8], where one can find the history of Maillet's determinant and Demjanenko matrix and many important results about them. In the same paper, Kučera showed a way of obtaining most of these determinant formulas in a unique fashion by means of the Stickelberger ideal. He also derived a nonvanishing determinant formula involving the relative class number by a modification of Ramachandra's construction of independent cyclotomic units.

In the function field case, some determinant formulas involving the relative class number and the real class number of cyclotomic function fields with prime power conductors are obtained by several authors (Rosen [9], Bae-Kang [3] and Jung-Ahn [6, 7]). Recently the authors gave determinant formulas for the real and relative class number of any subfield of a cyclotomic field with prime power conductor [2]. In this paper, by extending Kučera's idea [8, Lemma 2] to the function field case, we obtain several determinant formulas involving the real class number and the relative class number of any subfield of cyclotomic function fields with arbitrary conductor.

The layout of this paper is as follows. In Section 2, we derive a basic lemma (Lemma 2.1) which is essential for obtaining determinant formulas for class numbers. In Section 3, we state basic notation and results in cyclotomic theory over the rational function field which are needed later. Several determinant formulas involving the real class number and the relative class number of any subfield of cyclotomic function fields are obtained in subsection 3.1 and in subsection 3.2, respectively. Some of them may contain a zero factor. Adopting Kučera's idea [8] Example 4], we also give nonvanishing determinant formulas for class numbers. In

Received by the editor July 18, 2002 and, in revised form, October 1, 2003.

2000 Mathematics Subject Classification. Primary 11R58, 11R60.

This work was supported by grant No. R01-2002-000-00151-0 from the Basic Research Program of the Korea Science and Engineering Foundation. 
the final section, we provide several numerical examples by using these determinant formulas in Section 3.

\section{BASIC LEMMA}

Let $G$ be a finite abelian group with a fixed cyclic subgroup $J$. For any subset $M$ of $G$, let $s(M)=\sum_{\sigma \in M} \sigma \in \mathbb{Z}[G]$. Let $e^{+}=s(J) /|J|$ and $e^{-}=1-e^{+}$. For any $G$-submodule $H$ of $\mathbb{C}[G]$, we define two submodules $H^{+}, H^{-}$of $H$ as

$$
H^{+}=\left\{x \in H: e^{-} x=0\right\}, \quad H^{-}=\left\{x \in H: e^{+} x=0\right\} .
$$

Let $\widehat{G}$ be the set of all characters of $G$ with values in $\mathbb{C}$. For $\chi \in \widehat{G}$, we say that $\chi$ is real if $\chi(J)=\{1\}$ and $\chi$ is nonreal otherwise. Let $\widehat{G}^{+}$be the subset of $\widehat{G}$ consisting of real characters and $\widehat{G}^{-}=\widehat{G} \backslash \widehat{G}^{+}$. A character $\chi \in \widehat{G}$ induces a homomorphism $\rho_{\chi}: \mathbb{C}[G] \rightarrow \mathbb{C}$ defined as

$$
\rho_{\chi}\left(\sum_{\sigma \in G} c_{\sigma} \sigma\right)=\sum_{\sigma \in G} c_{\sigma} \chi(\sigma) .
$$

Let us denote by $\chi_{0}$ the trivial character of $G$.

Lemma 2.1. Let $C$ be a system of representatives of $G / J$. For any $\sigma \in G$, let $\tilde{\sigma} \in C$ be the unique element such that $\sigma \tilde{\sigma}^{-1} \in J$.

(i) For $\eta=\sum_{\sigma \in G} s_{\sigma} \sigma^{-1} \in \mathbb{C}[G]^{+}$, we have

$$
\operatorname{det}\left(s_{\sigma \tau}-s_{\sigma}: 1 \neq \sigma, \tau \in C\right)= \pm|J|^{(1-|C|)} \prod_{\chi 0 \neq \chi \in \widehat{G}^{+}} \rho_{\chi}(\eta) .
$$

(ii) For $\theta=\sum_{\sigma \in G} t_{\sigma} \sigma^{-1} \in \mathbb{C}[G]^{-}$, we have

$$
\operatorname{det}\left(t_{\sigma \tau}-t_{\sigma \tilde{\tau}}: \sigma, \tau \notin C\right)= \pm \prod_{\chi \in \widehat{G}^{-}} \rho_{\chi}(\theta) .
$$

Proof. For each $\chi \in \widehat{G}$, let $e_{\chi}=\frac{1}{|G|} \sum_{\sigma \in G} \chi(\sigma) \sigma^{-1}$ be the idempotent element associated with $\chi$. Let $\mathbb{C}[G]_{0}^{+}=\left\{\sum_{\sigma \in G} c_{\sigma} \sigma \in \mathbb{C}[G]^{+}: \sum_{\sigma \in G} c_{\sigma}=0\right\}$. For (i), we consider the linear transformation $S: \mathbb{C}[G]_{0}^{+} \rightarrow \mathbb{C}[G]_{0}^{+}$defined as multiplication by $\eta$. It is easy to see that both $X=\left\{\left(1-\sigma^{-1}\right) s(J): 1 \neq \sigma \in C\right\}$ and $\left\{e_{\chi}\right.$ : $\left.\chi \in \widehat{G}^{+}, \chi \neq \chi_{0}\right\}$ are $\mathbb{C}$-bases of $\mathbb{C}[G]_{0}^{+}$. The matrix of $S$ with respect to $X$ and $\left\{e_{\chi}: \chi \in \widehat{G}^{+}, \chi \neq \chi_{0}\right\}$ are $\left(|J|\left(s_{\sigma \tau^{-1}}-s_{\sigma}\right): 1 \neq \sigma, \tau \in C\right)$ and $\operatorname{diag}\left(\rho_{\chi}(\eta): \chi_{0} \neq\right.$ $\left.\chi \in \widehat{G}^{+}\right)$, respectively. Thus we have

$$
\operatorname{det}\left(|J|\left(s_{\sigma \tau^{-1}}-s_{\sigma}\right): 1 \neq \sigma, \tau \in C\right)=\prod_{\chi_{0} \neq \chi \in \widehat{G}^{+}} \rho_{\chi}(\eta) .
$$

Since $C^{\prime}=\left\{\sigma^{-1}: \sigma \in C\right\}$ is also a system of representatives of $G / J, X^{\prime}=$ $\{(1-\sigma) s(J): 1 \neq \sigma \in C\}$ is also a basis of $\mathbb{C}[G]_{0}^{+}$. The matrix of $S$ from $X^{\prime}$ to $X$ is given by $\left(|J|\left(s_{\sigma \tau}-s_{\sigma}\right): 1 \neq \sigma, \tau \in C\right)$. Since $X$ and $X^{\prime}$ are also $\mathbb{Z}$ bases of the free abelian group $\mathbb{Z}[G]_{0}^{+}$, the transition matrix from $X$ to $X^{\prime}$ has determinant \pm 1 . Therefore we get (i). For (ii), we consider the linear transformation $T: \mathbb{C}[G]^{-} \rightarrow \mathbb{C}[G]^{-}$defined as multiplication by $\theta$ and the following $\mathbb{C}$-bases of $\mathbb{C}[G]^{-},\left\{e_{\chi}: \chi \in \widehat{G}^{-}\right\}, Y=\left\{(1-j) \sigma^{-1}: 1 \neq j \in J, \sigma \in C\right\}$ and $Y^{\prime}=\{(1-j) \sigma:$ $1 \neq j \in J, \sigma \in C\}$. By similar arguments as for (i), we get (ii). 
When $J=\{1, j\}$, we have $t_{\sigma}+t_{j \sigma}=0$ and

$$
\tilde{\sigma}= \begin{cases}j \sigma & \text { if } \sigma \notin C, \\ \sigma & \text { if } \sigma \in C .\end{cases}
$$

Thus

$$
t_{\sigma \tau}-t_{\sigma \tilde{\tau}}= \begin{cases}2 t_{\sigma \tau} & \text { if } \tau \notin C, \\ 0 & \text { if } \tau \in C .\end{cases}
$$

From (2.2), we have

$$
\operatorname{det}\left(t_{\sigma \tau}: \sigma, \tau \notin C\right)= \pm \prod_{\chi \in \widehat{G}^{-}} \frac{1}{2} \rho_{\chi}(\theta) .
$$

Thus we may regard Lemma 2.1 (ii) as a generalization of [8, Lemma 2].

\section{Determinant FORMUlas FOR ClASS NUMBERS}

Let $\mathbb{A}=\mathbb{F}_{q}[T]$ be the ring of polynomials over a finite field $\mathbb{F}_{q}$ with $q$ elements, and let $\mathbb{F}_{q}(T)$ be the field of rational functions over $\mathbb{F}_{q}$. For each $N \in \mathbb{A}$ one uses the Carlitz module $\phi$ to construct a field extension $K_{N}$, called the $N$-th cyclotomic function field and its maximal real subfield $K_{N}^{+}$. It is known that the Galois group $G_{N}$ of $K_{N} / \mathbb{F}_{q}(T)$ is isomorphic to $(\mathbb{A} / N \mathbb{A})^{*}$, explicitly there is an isomorphism

$$
\Psi:(\mathbb{A} / N \mathbb{A})^{*} \rightarrow G_{N}, \quad A \bmod N \mathbb{A} \mapsto \sigma_{A},
$$

where $\sigma_{A}(\lambda)=\phi_{A}(\lambda)$ for any $N$-torsion point $\lambda$ of $\phi$. Moreover, $J=\operatorname{Gal}\left(K_{N} / K_{N}^{+}\right)$ $=\Psi\left(\mathbb{F}_{q}^{*}\right)$. Let $\Phi(N)$ be the order of $(\mathbb{A} / N \mathbb{A})^{*}$. Let $\mathbb{M}_{N}$ be the set of nonzero polynomials of degree less than $\operatorname{deg}(N)$ and relatively prime to $N$. Let $\mathbb{M}_{N}^{+}$be the subset of $\mathbb{M}_{N}$ consisting of monic polynomials and let $\mathbb{M}_{N}^{-}=\mathbb{M}_{N} \backslash \mathbb{M}_{N}^{+}$.

For any nonzero $A \in \mathbb{A}$, we define

$$
\operatorname{deg}_{N}(A)=\operatorname{deg}(\bar{A})
$$

where $\bar{A} \in \mathbb{M}_{N}$ is the unique polynomial such that $\bar{A} \equiv A \bmod N$. For any nonzero $A \in \mathbb{A}$, let $\operatorname{sgn}_{N}(A)$ be the leading coefficient of $\bar{A}$ and define

$$
\langle A\rangle_{N}= \begin{cases}1 & \text { if } \operatorname{sgn}_{N}(A)=1 \\ 0 & \text { otherwise }\end{cases}
$$

A character $\chi \in \widehat{G}_{N}$ may be viewed as a character of $(\mathbb{A} / N \mathbb{A})^{*}$, thus as a primitive Dirichlet character with conductor $F_{\chi}$, a monic polynomial. As a primitive Dirichlet character, it induces a function from $\mathbb{A}$ to $\mathbb{C}$ as follows; for any irreducible polynomial $P \in \mathbb{A}$, we define $\chi(P)=\chi\left(\left(P, K_{F_{\chi}} / \mathbb{F}_{q}(T)\right)\right)$ if $P \nmid F_{\chi}$ and $\chi(P)=0$ otherwise. Here $\left(P, K_{F_{\chi}} / \mathbb{F}_{q}(T)\right)$ denotes the Artin automorphism of $P$ in $K_{F_{\chi}}$.

Throughout the paper, we always assume that the finite abelian extension $k$ of $\mathbb{F}_{q}(T)$ is contained in some cyclotomic function field. By the conductor of $k$, we mean the monic polynomial $N \in \mathbb{A}$ such that $K_{N}$ is the smallest cyclotomic function field containing $k$. Let $k^{+}$be the maximal real subfield of $k$, i.e., $k^{+}=k \cap K_{N}^{+}$. Let $G=\operatorname{Gal}\left(k / \mathbb{F}_{q}(T)\right), G^{+}=\operatorname{Gal}\left(k^{+} / \mathbb{F}_{q}(T)\right)$ and $J_{k}=\operatorname{Gal}\left(k / k^{+}\right)$. We say that $k$ is a real (resp. nonreal) extension of $\mathbb{F}_{q}(T)$ if $k=k^{+}$(resp. $k \neq k^{+}$). For a divisor $F$ of $N$, we let $k_{F}=k \cap K_{F}$ and $k_{F}^{+}=k \cap K_{F}^{+}$.

We denote by $\operatorname{Div}^{0}(k)$ and $\mathcal{P}(k)$ the group of divisors of degree zero and the group of principal divisors of $k$, respectively. Then the divisor class number $h(k)$ 
of $k$ is defined to be the order of $\operatorname{Div}^{0}(k) / \mathcal{P}(k)$. It is well known that the divisor class number $h(k)$ of $k$ is divisible by the divisor class number $h\left(k^{+}\right)$of $k^{+}$, called the real divisor class number of $k$. The relative divisor class number of $k$, denoted by $h^{-}(k)$, is given by $h(k) / h\left(k^{+}\right)$.

A character $\chi \in \widehat{G}$ may be regarded as a character of $G_{N}$ which is trivial on $\operatorname{Gal}\left(K_{N} / k\right)$, thus as a primitive Dirichlet character. Let $d_{\chi}=\operatorname{deg}\left(F_{\chi}\right)$, and let $m_{\chi}(A)=\left(d_{\chi}-1-e\right)(q-1)-1$ if $A \in \mathbb{M}_{F_{\chi}}$ has degree $e$. Then there is a wellknown analytic class number formula for the real divisor class number $h\left(k^{+}\right)$[5] top of p. 408]:

$$
h\left(k^{+}\right)=(q-1)^{-r} \prod_{\substack{\chi \in \widehat{G}^{+} \\ \chi \neq \chi_{0}}} \sum_{A \in \mathbb{M}_{F_{\chi}}^{+}} m_{\chi}(A) \chi(A),
$$

where $r=\left[k^{+}: \mathbb{F}_{q}(T)\right]-1$. Let $F$ be a monic polynomial. For any $A \in \mathbb{M}_{F}$, $Z_{F}(s, A)$ denotes the partial zeta function associated with the class of $A$ in $\mathrm{Cl}(\mathbb{A})$. It is known [7, Lemma 3.1] that

$$
Z_{F}(0, A)= \begin{cases}(q-2) /(q-1) & \text { if } A \text { is monic, } \\ -1 /(q-1) & \text { otherwise. }\end{cases}
$$

As did Yin 11, Proposition 4], we have an analytic class number formula for the relative class number $h^{-}(k)$ when $k$ is a subextension of a cyclotomic function field:

$$
h^{-}(k)=\prod_{\chi \in \widehat{G}^{-}} L(0, \chi)=\prod_{\chi \in \widehat{G}^{-}} \sum_{A \in \mathbb{M}_{F_{\chi}}} Z_{F_{\chi}}(0, A) \chi(A),
$$

where $L(s, \chi)$ is the Artin $L$-function associated with the character $\chi$.

3.1. Real class numbers. Let $x=A / N \in \mathbb{F}_{q}(T) / \mathbb{A}$, where $A, N \in \mathbb{A}$ and $\operatorname{deg}(A)=e<d=\operatorname{deg}(N)$. Define $\varphi(x)=(q-1)(d-1-e)-1$. Then $\varphi$ is an ordinary distribution on $\mathbb{F}_{q}(T) / \mathbb{A}[4$, Proposition 3.1]. For any primitive Dirichlet character $\chi$, we let

$$
\varphi(\chi)=\sum_{A \in \mathbb{M}_{F \chi}} \varphi\left(A / F_{\chi}\right) \chi(A) .
$$

Let $F$ be any monic polynomial divisible by $F_{\chi}$. Then we have [4, Lemma 3.2] that

$$
\sum_{A \in \mathbb{M}_{F}} \varphi(A / F) \chi(A)=\varphi(\chi) \prod_{P \mid F}(1-\chi(P))
$$

where $P$ runs over all monic irreducible polynomials dividing $F$. For any real character $\chi$, we have

$$
\varphi(\chi)=(q-1) \sum_{A \in \mathbb{M}_{F_{\chi}}^{+}} \varphi\left(A / F_{\chi}\right) \chi(A),
$$

so the analytic class number formula (3.1) can be rewritten as

$$
h\left(k^{+}\right)=(q-1)^{-2 r} \prod_{\substack{\chi \in \widehat{G}^{+} \\ \chi \neq \chi_{0}}} \varphi(\chi) .
$$

For any $N \in \mathbb{A}$, by " $F \mid N$ " we mean that $F$ is a monic polynomial dividing $N$. For $A \in \mathbb{M}_{N}$, let $\sigma_{A}^{\prime}$ be the restriction of $\sigma_{A}$ on $k$. Now we state our basic determinant formula for $h\left(k^{+}\right)$. 
Theorem 3.1. Let $k$ be a finite abelian extension of $\mathbb{F}_{q}(T)$ with conductor $N$. Let $C$ be any system of representatives of $G / J_{k}$. Assume that we are given $\left\{a_{F, \sigma} \in \mathbb{Q}\right.$ : $1 \neq F \mid N, \sigma \in G\}$. For $\sigma \in G$, let $s_{\sigma}=\sum_{\substack{F \mid N \\ F \neq 1}} \sum_{A \in \mathbb{M}_{N}} a_{F, \sigma_{A}^{\prime} \sigma^{-1}} \varphi(A / F)$. Then we have

$$
\operatorname{det}\left(s_{\sigma \tau}-s_{\sigma}: 1 \neq \sigma, \tau \in C\right)= \pm\left|J_{k}\right|^{-r}(q-1)^{2 r} h\left(k^{+}\right) S_{k}^{+},
$$

where $S_{k}^{+}=\prod_{\substack{\chi \in \widehat{G}^{+} \\ \chi \neq \chi_{0}}} \sum_{\substack{F\left|N \\ F_{\chi}\right| F}} b_{F, \chi} \prod_{P \mid F}(1-\bar{\chi}(P))$ with $b_{F, \chi}=\frac{\Phi(N)}{\Phi(F)} \sum_{\sigma \in G} a_{F, \sigma} \chi(\sigma)$.

Proof. For each $F \mid N$ with $F \neq 1$, let $\eta_{F}=\sum_{A \in \mathbb{M}_{F}} \varphi(A / F) \sigma_{A}^{-1} \in \mathbb{Q}\left[G_{F}\right]$ and $\eta_{F}^{\prime}=\operatorname{cor}_{k / k_{F}} \operatorname{res}_{K_{F} / k_{F}}\left(\eta_{F}\right) \in \mathbb{Q}[G]$. By the definition of $\varphi$, it is obvious that $\eta_{F} \in \mathbb{Q}\left[G_{F}\right]^{+}$and so $\eta_{F}^{\prime} \in \mathbb{Q}[G]^{+}$. Here $\operatorname{res}_{K_{F} / k_{F}}$ and $\operatorname{cor}_{k / k_{F}}$ are the restriction and corestriction maps between group rings, respectively. We put

$$
\eta=\sum_{\substack{F \mid N \\ F \neq 1}} d_{F} \eta_{F}^{\prime}
$$

where $d_{F}=\left[K_{N}: k K_{F}\right] \sum_{\sigma \in G} a_{F, \sigma} \sigma \in \mathbb{Q}[G]$. Then we have

$$
\left[K_{N}: k K_{F}\right] \eta_{F}^{\prime}=\operatorname{res}_{K_{N} / k} \operatorname{cor}_{K_{N} / K_{F}}\left(\eta_{F}\right)=\sum_{A \in \mathbb{M}_{N}} \varphi(A / F) \sigma_{A}^{\prime}{ }^{-1} \in \mathbb{Q}[G]^{+},
$$

and so

$$
\eta=\sum_{\sigma \in G}\left(\sum_{\substack{F \mid N \\ F \neq 1}} \sum_{A \in \mathbb{M}_{N}} a_{F, \sigma_{A}^{\prime} \sigma^{-1}} \varphi(A / F)\right) \sigma^{-1}=\sum_{\sigma \in G} s_{\sigma} \sigma^{-1} .
$$

It follows, by Lemma 2.1 (i), that

$$
\operatorname{det}\left(s_{\sigma \tau}-s_{\sigma}: 1 \neq \sigma, \tau \in C\right)= \pm\left|J_{k}\right|^{-r} \prod_{\substack{\chi \in \widehat{G}^{+} \\ \chi \neq \chi_{0}}} \rho_{\chi}(\eta) .
$$

If $F_{\chi} \nmid F$, then $\rho_{\chi}\left(\eta_{F}^{\prime}\right)=0$ because $\chi$ is not trivial on $\operatorname{Gal}\left(k / k_{F}\right)$. Assume that $F_{\chi} \mid F$. Then by (3.4), we have

$$
\begin{aligned}
\rho_{\chi}\left(\eta_{F}^{\prime}\right) & =\left[k: k_{F}\right] \rho_{\chi}\left(\eta_{F}\right) \\
& =\left[k: k_{F}\right] \sum_{A \in \mathbb{M}_{F}} \varphi(A / F) \bar{\chi}(A)=\left[k: k_{F}\right] \varphi(\bar{\chi}) \prod_{P \mid F}(1-\bar{\chi}(P)),
\end{aligned}
$$

and so

$$
\begin{aligned}
\rho_{\chi}\left(d_{F} \eta_{F}^{\prime}\right) & =\left[K_{N}: k K_{F}\right] \sum_{\sigma \in G} a_{F, \sigma} \chi(\sigma)\left[k: k_{F}\right] \varphi(\bar{\chi}) \prod_{P \mid F}(1-\bar{\chi}(P)) \\
& =\varphi(\bar{\chi}) b_{F, \chi} \prod_{P \mid F}(1-\bar{\chi}(P)) .
\end{aligned}
$$

Thus we have

$$
\rho_{\chi}(\eta)=\varphi(\bar{\chi}) \sum_{\substack{F\left|N \\ F_{\chi}\right| F}} b_{F, \chi} \prod_{P \mid F}(1-\bar{\chi}(P)) .
$$

By substituting (3.9) into (3.7) and by the analytic class number formula (3.5), we get the result. 
In Theorem [3.1, $s_{\sigma}$ and $S_{k}$ depend on the choice of $\left\{a_{F, \sigma} \in \mathbb{Q}: 1 \neq F \mid N, \sigma \in\right.$ $G\}$. In the following we choose special values of $a_{F, \sigma}$ 's to get several determinant class number formulas.

For any monic irreducible polynomial $P \in \mathbb{A}$, we denote by $e_{P}$ and $f_{P}$ the ramification index and the residue class degree of $P$ in $k$, respectively. Let $g_{P}=$ $\left[k: \mathbb{F}_{q}(T)\right] /\left(e_{P} f_{P}\right)$, the number of primes in $k$ lying above $P$. We also use $e_{P}^{+}, f_{P}^{+}$ and $g_{P}^{+}$for the corresponding ones for $k^{+}$.

Lemma 3.2. Let $Q_{k}^{(+)}=\prod_{\substack{\chi \in \widehat{G}^{+} \\ \chi \neq \chi_{0}}} \prod_{P \mid N}(1-\bar{\chi}(P))$. Then we have

$$
Q_{k}^{(+)}= \begin{cases}\prod_{P \mid N} f_{P}^{+} & \text {if } g_{P}^{+}=1 \text { for all } P \mid N \\ 0 & \text { otherwise }\end{cases}
$$

In particular, if $k$ has prime power conductor, then we have $Q_{k}^{(+)}=1$.

Proof. Let $Q_{k}^{(+)}(z)=\prod_{\substack{x \in \widehat{G}^{+}+\chi_{0} \\ \chi \neq}} \prod_{P \mid N}(z-\bar{\chi}(P))$. Then the result follows from the fact that

$$
Q_{k}^{(+)}(z)=\prod_{P \mid N} \prod_{\substack{x \in \widehat{G}^{+} \\ \chi \neq \chi_{0}}}(z-\bar{\chi}(P))=\prod_{P \mid N} \frac{\left(z^{f_{P}^{+}}-1\right)^{g_{P}^{+}}}{z-1}
$$

where the last equality follows from [10, Theorem 3.7].

Take $a_{F, \sigma}=1$ for $F=N, \sigma=1$, and 0 otherwise. Then $s_{\sigma}=\sum_{\substack{A \in \mathbb{M}_{N} \\ \sigma_{A}^{\prime}=\sigma}} \varphi(A / N)$. Thus for any $\sigma, \tau \in G$

$$
\begin{aligned}
s_{\sigma \tau}-s_{\sigma} & =\sum_{\substack{A \in \mathbb{M}_{N} \\
\sigma_{A}^{\prime}=\sigma \tau}} \varphi(A / N)-\sum_{\substack{B \in \mathbb{M}_{N} \\
\sigma_{B}^{\prime}=\sigma}} \varphi(B / N) \\
& =-(q-1)\left(\sum_{\substack{A \in \mathbb{M}_{N} \\
\sigma_{A}^{\prime}=\sigma \tau}} \operatorname{deg}_{N}(A)-\sum_{\substack{B \in \mathbb{M}_{N} \\
\sigma_{B}^{\prime}=\sigma}} \operatorname{deg}_{N}(B)\right) .
\end{aligned}
$$

Moreover, we have $b_{F, \chi}=1$ for $F=N$ and 0 otherwise. Thus $S_{k}=Q_{k}^{(+)}$and so, by Theorem 3.1 , we get the following.

\section{Proposition 3.3.}

$$
\operatorname{det}\left(\sum_{\substack{A \in \mathbb{M}_{N} \\ \sigma_{A}^{\prime}=\sigma \tau}} \operatorname{deg}_{N}(A)-\sum_{\substack{B \in \mathbb{M}_{N} \\ \sigma_{B}^{\prime}=\sigma}} \operatorname{deg}_{N}(B): 1 \neq \sigma, \tau \in C\right)= \pm\left(\frac{q-1}{\left|J_{k}\right|}\right)^{r} h\left(k^{+}\right) Q_{k}^{(+)} .
$$

Now we assume that $k=K_{N}$. Taking $C=\left\{\sigma_{A}: A \in \mathbb{M}_{N}^{+}\right\}$as a system of representatives of $G_{N} / J$, we get the following corollary.

\section{Corollary 3.4.}

$$
\operatorname{det}\left(\operatorname{deg}_{N}(A B)-\operatorname{deg}_{N}(A): 1 \neq A, B \in \mathbb{M}_{N}^{+}\right)= \pm h\left(K_{N}^{+}\right) Q_{K_{N}}^{(+)}
$$

Unfortunately, when $Q_{k}^{(+)}=0$, formula (3.11) will not give any information. Thus, in this case, we need another choice of $a_{F, \sigma}$ to get the nonvanishing determinant formula. We will follow the idea of Kučera [8, Example 4]. Let $N=\prod_{i=1}^{s} P_{i}^{r_{i}}$ 
be the factorization of $N$ into monic irreducible polynomials. Let $S=\{1,2, \ldots, s\}$ and $N_{I}=\prod_{i \in I} P_{i}^{r_{i}}$ for any $I \subset S$. Put $c_{i}=-\Phi\left(P_{i}^{r_{i}}\right)^{-1}$ for each $1 \leq i \leq s$ and

$$
a_{F, \sigma}= \begin{cases}\prod_{i \notin I} c_{i} & \text { if } F=N_{I} \text { for some } I \subset S \text { and } \sigma=1, \\ 0 & \text { otherwise. }\end{cases}
$$

Then for any $\sigma \in G$, we have

$$
\begin{aligned}
s_{\sigma}=\sum_{\emptyset \neq I \subset S} \sum_{\substack{A \in \mathbb{M}_{N} \\
\sigma_{A}^{\prime}=\sigma}} \frac{(-1)^{s-|I|}}{\Phi\left(N / N_{I}\right)} \varphi\left(A / N_{I}\right) & =\sum_{\emptyset \neq I \subset S} \frac{(-1)^{s-|I|}}{\Phi\left(N / N_{I}\right)} \sum_{\substack{A \in \mathbb{M}_{N} \\
\sigma_{A}^{\prime}=\sigma}} \varphi\left(A / N_{I}\right) \\
& =\sum_{I \subsetneq S} \frac{(-1)^{|I|}}{\Phi\left(N_{I}\right)} \sum_{\substack{A \in \mathbb{M}_{N} \\
\sigma_{A}^{\prime}=\sigma}} \varphi\left(A /\left(N / N_{I}\right)\right) .
\end{aligned}
$$

Thus

$$
s_{\sigma \tau}-s_{\sigma}=-(q-1) \sum_{I \subsetneq S}(-1)^{|I|} \Phi\left(N_{I}\right)^{-1}\left(\sum_{\substack{A \in \mathbb{M}_{N} \\ \sigma_{A}^{\prime}=\sigma \tau}} \operatorname{deg}_{N / N_{I}}(A)-\sum_{\substack{B \in \mathbb{M}_{N} \\ \sigma_{B}^{\prime}=\sigma}} \operatorname{deg}_{N / N_{I}}(B)\right) \text {, }
$$

for any $\sigma, \tau \in G$. We note that, in this case,

$$
S_{k}=\prod_{\substack{\chi \in \widehat{G}^{+} \\ \chi \neq \chi_{0}}} \sum_{\substack{\left.I \subset S \\ F_{\chi}\right|_{I}}} \frac{\Phi(N)}{\Phi\left(N_{I}\right)} \prod_{i \notin I} c_{i} \prod_{i \in I}\left(1-\bar{\chi}\left(P_{i}\right)\right)=\prod_{i \in S} \prod_{\substack{\chi_{0} \neq \chi \in \widehat{G}^{+} \\ P_{i}+F_{\chi}}}\left(-\bar{\chi}\left(P_{i}\right)\right)= \pm 1 .
$$

Define

$$
\mathbb{S}_{k}=\left(\sum_{I \subsetneq S}(-1)^{|I|} \Phi\left(N_{I}\right)^{-1}\left(\sum_{\substack{A \in \mathbb{M}_{N} \\ \sigma_{A}^{\prime}=\sigma \tau}} \operatorname{deg}_{N / N_{I}}(A)-\sum_{\substack{B \in \mathbb{M}_{N} \\ \sigma_{B}^{\prime}=\sigma}} \operatorname{deg}_{N / N_{I}}(B)\right): 1 \neq \sigma, \tau \in C\right) \text {. }
$$

Then from Theorem 3.1 we get the following nonvanishing determinant class number formula for $h\left(k^{+}\right)$.

Proposition 3.5. $\operatorname{det}\left(\mathbb{S}_{k}\right)= \pm\left((q-1) /\left|J_{k}\right|\right)^{r} h\left(k^{+}\right)$.

In particular, if $k=K_{N}$, the matrix $\mathbb{S}_{K_{N}}$ is given as

$$
\mathbb{S}_{K_{N}}=\left(\sum_{I \subsetneq S}(-1)^{|I|} \Phi\left(N_{I}\right)^{-1}\left(\operatorname{deg}_{N / N_{I}}(A B)-\operatorname{deg}_{N / N_{I}}(A)\right): 1 \neq A, B \in \mathbb{M}_{N}^{+}\right) .
$$

3.2. Relative class numbers. For a monic polynomial $F \in \mathbb{A}$, let

$$
\theta_{F}=\sum_{A \in \mathbb{M}_{F}} Z_{F}(0, A) \sigma_{A}^{-1} \in \mathbb{Q}\left[G_{F}\right]
$$

Recall that $N$ is the conductor of $k$. For any $F \mid N$ with $F \neq 1$, we define

$$
\theta_{F}^{\prime}=\operatorname{cor}_{k / k_{F}} \operatorname{res}_{K_{F} / k_{F}}\left(\theta_{F}\right) \in \mathbb{Q}[G] .
$$

By (3.2), it is obvious that $\theta_{F} \in \mathbb{Q}[G]^{-}$and so $\theta_{F}^{\prime} \in \mathbb{Q}[G]^{-}$. Then we have

$$
\left[K_{N}: k K_{F}\right] \theta_{F}^{\prime}=\operatorname{res}_{K_{N} / k} \operatorname{cor}_{K_{N} / K_{F}}\left(\theta_{F}\right)=\sum_{A \in \mathbb{M}_{N}} Z_{F}(0, A) \sigma_{A}^{\prime-1} \in \mathbb{Q}[G]^{-}
$$


If $F_{\chi} \nmid F$, then $\rho_{\chi}\left(\theta_{F}^{\prime}\right)=0$ because $\chi$ is not trivial on $\operatorname{Gal}\left(k / k_{F}\right)$. Assume that $F_{\chi} \mid F$. As in [10, Lemma 8.7],

$$
\rho_{\chi}\left(\theta_{F}^{\prime}\right)=\left[k: k_{F}\right] \rho_{\chi}\left(\theta_{F}\right)=\left[k: k_{F}\right] L(0, \bar{\chi}) \prod_{P \mid F}(1-\bar{\chi}(P)),
$$

where $P$ runs over all monic irreducible polynomials dividing $F$.

Theorem 3.6. Let $k$ be a finite abelian extension of $\mathbb{F}_{q}(T)$ with conductor $N$. Let $C$ be any system of representatives of $G / J_{k}$. Assume that we are given $\left\{a_{F, \sigma} \in \mathbb{Q}\right.$ : $1 \neq F \mid N, \sigma \in G\}$. For any $\sigma \in G$, we let

$$
t_{\sigma}=\sum_{\substack{F \mid N \\ F \neq 1}} \sum_{A \in \mathbb{M}_{N}} Z_{F}(0, A) a_{F, \sigma_{A}^{\prime} \sigma^{-1}}
$$

Then we have

$$
\operatorname{det}\left(t_{\sigma \tau}-t_{\sigma \tilde{\tau}}: \sigma, \tau \notin C\right)= \pm h^{-}(k) S_{k}^{-},
$$

where $S_{k}^{-}=\prod_{\chi \in \widehat{G}^{-}} \sum_{\substack{F\left|N \\ F_{\chi}\right| F}} b_{F, \chi} \prod_{P \mid F}(1-\bar{\chi}(P))$ with $b_{F, \chi}=\frac{\Phi(N)}{\Phi(F)} \sum_{\sigma \in G} a_{F, \sigma} \chi(\sigma)$.

Proof. We consider $\theta=\sum_{F \mid N, F \neq 1} d_{F} \theta_{F}^{\prime}$. Then, from (3.14), we have

$$
\theta=\sum_{\sigma \in G} \sum_{\substack{F \mid N \\ F \neq 1}} \sum_{A \in \mathbb{M}_{N}} Z_{F}(0, A) a_{F, \sigma} \sigma_{A}^{\prime-1} \sigma=\sum_{\sigma \in G} t_{\sigma} \sigma^{-1} .
$$

By Lemma 2.1(ii), we have

$$
\operatorname{det}\left(t_{\sigma \tau}-t_{\sigma \tilde{\tau}}: \sigma, \tau \notin C\right)= \pm \prod_{\chi \in \widehat{G}^{-}} \rho_{\chi}(\theta)= \pm \prod_{\chi \in \widehat{G}^{-}} \sum_{\substack{F \mid N \\ F \neq 1}} \rho_{\chi}\left(\theta_{F}^{\prime}\right) \rho_{\chi}\left(d_{F}\right) .
$$

Since $\chi\left(d_{F}\right)=\left[K_{N}: k K_{F}\right] \sum_{\sigma \in G} a_{F, \sigma} \chi(\sigma)$, by (3.15), we have

$$
\rho_{\chi}\left(\theta_{F}^{\prime}\right) \rho_{\chi}\left(d_{F}\right)= \begin{cases}L(0, \bar{\chi}) b_{F, \chi} \prod_{P \mid F}(1-\bar{\chi}(P)) & \text { if } F_{\chi} \mid F \\ 0 & \text { otherwise. }\end{cases}
$$

Therefore, by substituting (3.18) into (3.17) and by the analytic class number formula (3.3), we have

$$
\begin{aligned}
\operatorname{det}\left(t_{\sigma \tau}-t_{\sigma \tilde{\tau}}: \sigma, \tau \notin C\right) & = \pm \prod_{\chi \in \widehat{G}^{-}} \sum_{\substack{F\left|N \\
F_{\chi}\right| F}} L(0, \bar{\chi}) b_{F, \chi} \prod_{P \mid F}(1-\bar{\chi}(P)) \\
& = \pm \prod_{\chi \in \widehat{G}^{-}} L(0, \bar{\chi}) \prod_{\chi \in \widehat{G}^{-}} \sum_{\substack{F\left|N \\
F_{\chi}\right| F}} b_{F, \chi} \prod_{P \mid F}(1-\bar{\chi}(P)) \\
& = \pm h^{-}(k) \prod_{\chi \in \widehat{G}^{-}} \sum_{\substack{F\left|N \\
F_{\chi}\right| F}} b_{F, \chi} \prod_{P \mid F}(1-\bar{\chi}(P)) . \quad \square
\end{aligned}
$$

As for the real class number case, we choose special values of $a_{F, \sigma}$ to get several determinant class number formulas.

Lemma 3.7. Let $Q_{k}^{(-)}=\prod_{\chi \in \widehat{G}^{-}} \prod_{P \mid N}(1-\bar{\chi}(P))$. Then we have

$$
Q_{k}^{(-)}= \begin{cases}\prod_{P \mid N}\left(f_{P} / f_{P}^{+}\right)^{g_{P}} & \text { if } g_{P}=g_{P}^{+} \text {for all } P \mid N, \\ 0 & \text { otherwise. }\end{cases}
$$

In particular, if $k$ has prime power conductor, then we have $Q_{k}^{(-)}=1$. 
Proof. Let $Q_{k}(z)=\prod_{\chi_{0} \neq \chi \in \widehat{G}} \prod_{P \mid N}(z-\bar{\chi}(P))$ and $Q_{k}^{(-)}(z)=Q_{k}(z) / Q_{k}^{(+)}(z)$. By [10, Theorem 3.7], we have $Q_{k}(z)=\prod_{P \mid N}\left(z^{f_{P}}-1\right)^{g_{P}} /(z-1)$ and so

$$
Q_{k}^{(-)}(z)=\prod_{P \mid N} \frac{\left(z^{f_{P}}-1\right)^{g_{P}}}{\left(z^{f_{P}^{+}}-1\right)^{g_{P}^{+}}}
$$

By evaluating $Q_{k}^{(-)}(z)$ at $z=1$, we get the result.

For any $A \in \mathbb{M}_{N}$, we let $\tilde{A}=A / \operatorname{sgn}(A) \in \mathbb{M}_{N}^{+}$.

Proposition 3.8. Let $f: J_{k} \rightarrow \mathbb{C}$ be any map. For any $\sigma \in G$, we let

$$
t_{\sigma}=\sum_{\alpha \in J_{k}} \sum_{\substack{A \in \mathbb{M}_{N} \\ \sigma_{A}^{\prime}=\alpha \sigma}} Z_{N}(0, A) f(\alpha) ;
$$

that is, we take $a_{F, \sigma}=f(\sigma)$ if $F=N, \sigma \in J_{k}$ and 0 otherwise. Then we have

$$
\operatorname{det}\left(t_{\sigma \tau}-t_{\sigma \tilde{\tau}}: \sigma, \tau \notin C\right)= \pm h^{-}(k) A_{f}^{\left[k^{+}: \mathbb{F}_{q}(T)\right]} Q_{k}^{(-)},
$$

where $A_{f}=\operatorname{det}\left(f\left(\alpha \beta^{-1}\right)-f(\alpha): 1 \neq \alpha, \beta \in J_{k}\right)$. In particular, if $k=K_{N}$, then we have

$$
\operatorname{det}\left(f\left(\operatorname{sgn}_{N}(A B)\right)-f\left(\operatorname{sgn}_{N}(A \tilde{B})\right): A, B \in \mathbb{M}_{N}^{-}\right)= \pm h^{-}\left(K_{N}\right) A_{f}^{\left[K_{N}^{+}: \mathbb{F}_{q}(T)\right]} Q_{K_{N}}^{(-)} .
$$

Proof. Define $a_{F, \sigma}=f(\sigma)$ if $F=N, \sigma \in J_{k}$ and $a_{F, \sigma}=0$ otherwise. Then we have

$$
t_{\sigma}=\sum_{A \in \mathbb{M}_{N}} Z_{N}(0, A) a_{N, \sigma_{A}^{\prime} \sigma^{-1}}=\sum_{\alpha \in J_{k}} \sum_{\substack{A \in \mathbb{M}_{N} \\ \sigma_{A}^{\prime}=\alpha \sigma}} Z_{N}(0, A) f(\alpha) .
$$

We note that $b_{F, \chi}=\sum_{\alpha \in J_{k}} f(\alpha) \chi(\alpha)$ if $F=N$ and 0 otherwise. Thus

$$
\begin{aligned}
\prod_{\chi \in \widehat{G}^{-}} b_{N, \chi} & =\prod_{\chi \in \widehat{G}^{-}} \sum_{\alpha \in J_{k}} f(\alpha) \chi(\alpha)=\left(\prod_{\substack{\chi \in \widehat{J}_{k} \\
\chi \neq \chi_{0}}} \sum_{\alpha \in J_{k}} f(\alpha) \chi(\alpha)\right)^{\left[k^{+}: \mathbb{F}_{q}(T)\right]} \\
& =\left(\operatorname{det}\left(f\left(\alpha \beta^{-1}\right)-f(\alpha): 1 \neq \alpha, \beta \in J_{k}\right)\right)^{\left[k^{+}: \mathbb{F}_{q}(T)\right]},
\end{aligned}
$$

where the last equality follows from the Dedekind determinant formula [10, Lemma 5.26 (b)]. Therefore from Theorem 3.6, we prove (3.20). Now assume that $k=K_{N}$. For each $A \in \mathbb{M}_{N}$, we have

$$
t_{\sigma_{A}}=\sum_{\alpha \in \mathbb{F}_{q}^{*}} Z_{N}\left(0, \alpha^{-1} A\right) f(\alpha)=f\left(\operatorname{sgn}_{N}(A)\right)-\sum_{\alpha \in \mathbb{F}_{q}^{*}} \frac{f(\alpha)}{q-1} .
$$

Thus $t_{\sigma_{A} \sigma_{B}}-t_{\sigma_{A} \tilde{\sigma}_{B}}=f\left(\operatorname{sgn}_{N}(A B)\right)-f\left(\operatorname{sgn}_{N}(A \tilde{B})\right)$, for any $A, B \in \mathbb{M}_{N}$. This completes the proof of (3.21).

Now let us choose a specific map $f: J_{k} \rightarrow\{0,1\}$ by $f(\alpha)=1$ if $\alpha=1$ and 0 otherwise. Then we have the following generalized Demjanenko matrix for the relative class number of $k$.

Corollary 3.9. Let $t_{\sigma}=\sum_{A \in \mathbb{M}_{N}, \sigma_{A}^{\prime}=\sigma} Z_{N}(0, A)$. Then we have

$$
\operatorname{det}\left(t_{\sigma \tau}-t_{\sigma \bar{\tau}}: \sigma, \tau \notin C\right)= \pm h^{-}(k) Q_{k}^{(-)} .
$$


When $k=K_{N}$, let $\mathcal{O}_{K_{N}}$ be the integral closure of $\mathbb{A}$ in $K_{N}$ and let $h^{-}\left(\mathcal{O}_{K_{N}}\right)$ be the relative ideal class number of $\mathcal{O}_{K_{N}}$. Then we have

$$
\operatorname{det}\left(\langle A B\rangle_{N}: A, B \in \mathbb{M}_{N}^{-}\right)= \pm \begin{cases}h^{-}\left(\mathcal{O}_{K_{N}}\right) & \text { if } N \text { is a prime power } \\ h^{-}\left(\mathcal{O}_{K_{N}}\right) Q_{K_{N}}^{(-)} /(q-1) & \text { otherwise. }\end{cases}
$$

Proof. Since $b_{F, \chi}=1$ if $F=N$ and 0 otherwise, the first statement follows from (3.20). Assume that $k=K_{N}$. Since $f\left(\operatorname{sgn}_{N}(A)\right)=\langle A\rangle_{N}$, we have

$$
\operatorname{det}\left(\langle A B\rangle_{N}-\langle A \tilde{B}\rangle_{N}: A, B \in \mathbb{M}_{N}^{-}\right)= \pm h^{-}\left(K_{N}\right) Q_{K_{N}}^{(-)} .
$$

From [11 Lemma 3], we have

$$
h^{-}\left(K_{N}\right)= \begin{cases}h^{-}\left(\mathcal{O}_{K_{N}}\right)(q-1)^{\left[K_{N}^{+}: \mathbb{F}_{q}(T)\right]-1} & \text { if } N \text { is a prime power, } \\ h^{-}\left(\mathcal{O}_{K_{N}}\right)(q-1)^{\left[K_{N}^{+}: \mathbb{F}_{q}(T)\right]-2} & \text { otherwise. }\end{cases}
$$

In [6, Proposition 2.6], it is shown that

$\operatorname{det}\left(\langle A B\rangle_{N}-\langle A \tilde{B}\rangle_{N}: A, B \in \mathbb{M}_{N}^{-}\right)= \pm(q-1)^{\left[K_{N}^{+}: \mathbb{F}_{q}(T)\right]-1} \operatorname{det}\left(\langle A B\rangle_{N}: A, B \in \mathbb{M}_{N}^{-}\right)$, when $N$ is a prime power. It is easy to see that the proof of [6, Proposition 2.6] also holds for an arbitrary polynomial $N$. We note that $Q_{K_{N}}^{(-)}$is 1 if $N$ is a prime power. Therefore we get the result.

We give another matrix whose determinant is also related to $h^{-}(k)$. Let $j$ be a fixed generator of $J_{k}$. We define $f: J_{k} \rightarrow\left\{0, \ldots,\left|J_{k}\right|-1\right\}$ as $\alpha=j^{f(\alpha)}$ for any $\alpha \in J_{k}$. When $k=K_{N}$, we let $\overline{\operatorname{sgn}}_{N}(A)=f\left(\operatorname{sgn}_{N}(A)\right)$ for $A \in \mathbb{A}$. Then we have the following.

Corollary 3.10. Assume that $q>2$. For each $\sigma \in G$, we let

$$
t_{\sigma}=\sum_{i=0}^{\left|J_{k}\right|-1}\left(\sum_{\substack{A \in \mathbb{M}_{N} \\ \sigma_{A}^{\prime} j^{i^{2} \sigma}}} Z_{N}(0, A)\right) i
$$

Then we have

$$
\operatorname{det}\left(t_{\sigma \tau}-t_{\sigma \tilde{\tau}}: \sigma, \tau \notin C\right)= \pm h^{-}(k)\left|J_{k}\right|^{\left(\left|J_{k}\right|-2\right)\left[k^{+}: \mathbb{F}_{q}(T)\right]} Q_{k}^{(-)} .
$$

For $k=K_{N}$,

$$
\operatorname{det}\left(\overline{\operatorname{sgn}}_{N}(A B)-\overline{\operatorname{sgn}}_{N}(A \tilde{B}): A, B \in \mathbb{M}_{N}^{-}\right)= \pm h^{-}\left(K_{N}\right)(q-1)^{\frac{q-3}{q-1} \Phi(N)} Q_{K_{N}}^{(-)} .
$$

Proof. We note that

$$
\begin{aligned}
\operatorname{det}\left(f\left(\alpha \beta^{-1}\right)-f(\alpha): 1 \neq \alpha, \beta \in J_{k}\right) & =\prod_{\chi \in \widehat{J}_{k}, \chi \neq 1} \sum_{\alpha \in J_{k}} f(\alpha) \chi(\alpha) \\
& =\prod_{\chi \in \widehat{J}_{k}, \chi \neq 1} \sum_{i=0}^{\left|J_{k}\right|-1} i \chi(j)^{i} .
\end{aligned}
$$

Here $\chi(j)$ ranges over all nontrivial $\left|J_{k}\right|$-th roots of unity as $\chi$ runs over $\widehat{J}_{k} \backslash\{1\}$. Consider $g(X)=\left(X^{\left|J_{k}\right|}-1\right) /(X-1)=X^{\left|J_{k}\right|-1}+\cdots+1$. Then the discriminant of $g$ is $\pm\left|J_{k}\right|^{\left|J_{k}\right|-2}$. Thus we have

$$
\operatorname{det}\left(f\left(\alpha \beta^{-1}\right)-f(\alpha): 1 \neq \alpha, \beta \in J_{k}\right)=\prod_{\zeta^{\left|J_{k}\right|}=1, \zeta \neq 1} \zeta g^{\prime}(\zeta)= \pm\left|J_{k}\right|^{\left|J_{k}\right|-2} .
$$

Now the results follow from Proposition 3.8 
Finally, we also need a nonvanishing determinant class number formula for $h^{-}(k)$, in the case that $Q_{k}^{(-)}=0$. We follow the same notations and definitions for $S, N_{I}$ and $a_{F, \sigma}$ 's as in the real class number case. Then we have

$$
\begin{aligned}
t_{\sigma} & =\sum_{\emptyset \neq I \subset S} \sum_{\substack{A \in \mathbb{M}_{N} \\
\sigma_{A}^{\prime}=\sigma}}(-1)^{s-|I|} \Phi\left(N / N_{I}\right)^{-1} Z_{N_{I}}(0, A) \\
& =\sum_{I \subsetneq S}(-1)^{|I|} \Phi\left(N_{I}\right) \sum_{\substack{A \in \mathbb{M}_{N} \\
\sigma_{A}^{\prime}=\sigma}} Z_{N / N_{I}}(0, A) .
\end{aligned}
$$

Thus

$$
\begin{aligned}
t_{\sigma \tau}-t_{\sigma \tilde{\tau}} & =\sum_{I \subsetneq S}(-1)^{|I|} \Phi\left(N_{I}\right)^{-1}\left(\sum_{\substack{A \in \mathbb{M}_{N} \\
\sigma_{A}^{\prime}=\sigma \tau}} Z_{N / N_{I}}(0, A)-\sum_{\substack{B \in \mathbb{M}_{N} \\
\sigma_{B}^{\prime}=\sigma \tilde{\tau}}} Z_{N / N_{I}}(0, B)\right) \\
& =\sum_{I \subsetneq S}(-1)^{|I|} \Phi\left(N_{I}\right)^{-1}\left(\sum_{\substack{A \in \mathbb{M}_{N} \\
\sigma_{A}^{\prime}=\sigma \tau}}\langle A\rangle_{N / N_{I}}-\sum_{\substack{B \in \mathbb{M}_{N} \\
\sigma_{B}^{\prime}=\sigma \tilde{\tau}}}\langle B\rangle_{N / N_{I}}\right) .
\end{aligned}
$$

As in (3.13), we have

$$
\prod_{\chi \in \hat{G}^{-}} \sum_{\substack{F \mid N \\ F_{\chi} \backslash F}} b_{F, \chi} \prod_{P \mid F}(1-\bar{\chi}(P))=\prod_{\chi \in \hat{G}^{-}} \sum_{\substack{\left.I \in S \\ F_{\chi}\right|_{I}}}(-1)^{s-|I|} \prod_{i \in I}\left(1-\bar{\chi}\left(P_{i}\right)\right)= \pm 1 .
$$

Define

$$
\mathbb{T}_{k}=\left(\sum_{I \subsetneq S}(-1)^{|I|} \Phi\left(N_{I}\right)^{-1}\left(\sum_{\substack{A \in \mathbb{M}_{N} \\ \sigma_{A}^{\prime}=\sigma \tau}}\langle A\rangle_{N / N_{I}}-\sum_{\substack{B \in \mathbb{M}_{N} \\ \sigma_{B}^{\prime}=\sigma \tilde{\tau}}}\langle B\rangle_{N / N_{I}}\right): \sigma, \tau \notin C\right) .
$$

Then from Theorem 3.6, we have the following.

Proposition 3.11. $\operatorname{det}\left(\mathbb{T}_{k}\right)= \pm h^{-}(k)$.

When $k=K_{N}$, the matrix $\mathbb{T}_{K_{N}}$ is given as

$$
\mathbb{T}_{K_{N}}=\left(\sum_{I \subsetneq S}(-1)^{|I|} \Phi\left(N_{I}\right)^{-1}\left(\langle A B\rangle_{N / N_{I}}-\langle A \tilde{B}\rangle_{N / N_{I}}\right): A, B \in \mathbb{M}_{N}^{-}\right) .
$$

\section{Some Numerical EXAMPLES}

Example 4.1. Assume that $q=3$ and $k=K_{P Q}$ with $P=T$ and $Q=T^{2}+T+2$. Then $\mathbb{M}_{P Q}^{+}=\left\{1, T+1, T+2, T^{2}+1, T^{2}+2, T^{2}+T+1, T^{2}+2 T+1, T^{2}+2 T+2\right\}$ and $\mathbb{M}_{P Q}^{-}=\left\{2,2 T+1,2 T+2,2 T^{2}+1,2 T^{2}+2,2 T^{2}+T+1,2 T^{2}+T+2,2 T^{2}+2 T+2\right\}$. We use $\mathbb{M}_{P Q}^{+}$and $\mathbb{M}_{P Q}^{-}$in this ordering for matrices in this example. Then the matrix in (3.12) with $N=P Q$ is given as

$$
\left(\begin{array}{rrrrrrr}
1 & 1 & 0 & -1 & 1 & 1 & 1 \\
1 & 1 & 1 & 1 & 1 & -1 & 0 \\
-1 & 0 & 0 & 0 & -1 & 0 & -2 \\
-2 & 0 & 0 & -1 & 0 & -1 & 0 \\
0 & 0 & -1 & 0 & -2 & -1 & 0 \\
0 & -2 & 0 & -1 & -1 & 0 & 0 \\
0 & -1 & -2 & 0 & 0 & 0 & -1
\end{array}\right)
$$


An elementary calculation shows that $g_{P}^{+}=g_{Q}^{+}=g_{P}=g_{Q}=1$ and so $Q_{K_{N}}^{(+)}=$ $Q_{K_{N}}^{(-)}=4$. Thus we have $h\left(K_{N}^{+}\right)=2 \cdot 17$. The matrix in (3.22) is given as

$$
\left(\begin{array}{llllllll}
1 & 1 & 1 & 1 & 1 & 1 & 1 & 1 \\
1 & 1 & 1 & 1 & 1 & 1 & 0 & 0 \\
1 & 1 & 1 & 0 & 0 & 0 & 0 & 1 \\
1 & 1 & 0 & 0 & 0 & 1 & 0 & 1 \\
1 & 1 & 0 & 0 & 1 & 0 & 0 & 1 \\
1 & 1 & 0 & 1 & 0 & 0 & 0 & 1 \\
1 & 0 & 0 & 0 & 0 & 0 & 1 & 0 \\
1 & 0 & 1 & 1 & 1 & 1 & 0 & 1
\end{array}\right) .
$$

Thus we have $h^{-}\left(\mathcal{O}_{K_{N}}\right)=5$.

Example 4.2. Assume that $q=3$ and $k=K_{P Q}$ with $P=T$ and $Q=T^{2}+1$. In this case it is easy to check that $g_{P}^{+}=g_{P}=g_{Q}=2$ and $g_{Q}^{+}=1$. Thus $Q_{K_{P Q}}^{(+)}=Q_{K_{P Q}}^{(-)}=0$ and we cannot compute the real class number and the relative class number of $K_{P Q}$ from (3.12) and (3.22), respectively. We compute the class numbers of $K_{P Q}$ from the nonvanishing formulas in Propositions 3.5 and 3.11 Then we have $h\left(K_{P Q}^{+}\right)=2^{2} \cdot 3^{2}$ and $h^{-}\left(\mathcal{O}_{K_{P Q}}\right)=2^{2}$.

Example 4.3. Assume $q=3$ and $k=K_{N}$ with $N=T\left(T^{2}+1\right)\left(T^{2}+T+2\right)$. In this case both $Q_{K_{N}}^{(+)}$and $Q_{K_{N}}^{(-)}$are zero. Using Propositions 3.5 and 3.11 , we compute that

$$
h\left(K_{N}^{+}\right)=2^{42} \cdot 3^{8} \cdot 5^{2} \cdot 7^{2} \cdot 17^{6} \cdot 113 \cdot 241^{2} \cdot 257 \cdot 281 \cdot 433
$$

and

$$
h^{-}\left(\mathcal{O}_{K_{N}}\right)=2^{13} \cdot 3^{6} \cdot 5^{6} \cdot 13 \cdot 17^{2} \cdot 41^{2} \cdot 137 \cdot 193 \cdot 241 \cdot 281 .
$$

Example 4.4. When $q=3$ and $N=T^{2}\left(T^{2}+1\right)$, we have

$$
h\left(K_{N}^{+}\right)=2^{8} \cdot 3^{6} \cdot 11^{2} \cdot 73^{2}, \quad h^{-}\left(\mathcal{O}_{K_{N}}\right)=2^{8} \cdot 13 \cdot 457 .
$$

When $q=3$ and $N=T^{2}\left(T^{2}+T+2\right)$, we have

$$
h\left(K_{N}^{+}\right)=2 \cdot 17 \cdot 19 \cdot 337 \cdot 409 \cdot 733, \quad h^{-}\left(\mathcal{O}_{K_{N}}\right)=5^{3} \cdot 7^{3} \cdot 37 .
$$

When $q=5$ and $N=T^{2}(T+1)$, we have

$$
h\left(K_{N}^{+}\right)=41 \cdot 71 \cdot 101, \quad h^{-}\left(\mathcal{O}_{K_{N}}\right)=2^{4} \cdot 5 \cdot 11 \cdot 41 \cdot 1301 \cdot 11981 \cdot 15601 \cdot 57301 .
$$

Example 4.5. Table 1 gives the real and relative class numbers of $K_{N}$ for $q=3$ and $N=T^{n}(T+1)$ with $n=1,2,3,4$.

TABle 1. $q=3, N=T^{n}(T+1)$

\begin{tabular}{|c|c|c|}
\hline$n$ & $h\left(K_{N}^{+}\right)$ & $h^{-}\left(\mathcal{O}_{K_{N}}\right)$ \\
\hline 1 & 1 & 1 \\
\hline 2 & 7 & 1 \\
\hline 3 & $2^{6} \cdot 7^{5} \cdot 13$ & $2^{2} \cdot 7^{2} \cdot 19$ \\
\hline 4 & $2^{6} \cdot 7^{5} \cdot 13 \cdot 19^{2} \cdot 37 \cdot 109^{2} \cdot 127$ & $2^{2} \cdot 7^{2} \cdot 19 \cdot 37^{2} \cdot 127 \cdot 181$ \\
& $\cdot 307^{2} \cdot 1009 \cdot 10531 \cdot 270379$ & $\cdot 307 \cdot 379 \cdot 523 \cdot 9091$ \\
\hline
\end{tabular}




\section{ACKNOWLEDGMENTS}

The authors would like to express their sincere gratitude to the referee for his careful reading of the manuscript and suggestions on writing of the paper.

\section{REFERENCES}

[1] J. Ahn, S. Bae and H. Jung, Cyclotomic units and Stickelberger ideals of global function fields. Trans. Amer. Math. Soc. 355 (2003), no. 5, 1803-1818.

[2] S. Bae, H. Jung and J. Ahn, Class numbers of some abelian extensions of rational function fields. Math. Comp. 73 (2004), no. 245, 377-386.

[3] S. Bae and P. Kang, Class numbers of cyclotomic function fields. Acta Arith. 102 (2002), no. 3, 251-259. MR 2002m:11098

[4] S. Galovich and M. Rosen, Units and class group in cyclotomic function fields. J. Number Theory 14 (1982), 156-184. MR 84b:12008

[5] F. Harrop, Circular units of function fields. Trans. Amer. Math. Soc. 341 (1994), 405-421. MR 94c:11106

[6] H. Jung and J. Ahn, On the relative class number of cyclotomic function fields. Acta Arith. 107 (2003), no. 1, 91-101. MR 2003j:11143

[7] H. Jung and J. Ahn, Demjanenko matrix and recursion formula for relative class number over function fields. J. Number Theory 98 (2003), no. 1, 55-66. MR 2003i:11170

[8] R. Kučera, Formulae for the relative class number of an imaginary abelian field in the form of a determinant. Nagoya Math. J. 163 (2001), 167-191. MR 2002j:11129

[9] M. Rosen, A note on the relative class number in function fields. Proc. Amer. Math. Soc. 125 (1997), no. 5, 1299-1303. MR 97g:11133

[10] L. Washington, Introduction to cyclotomic fields. Second edition. GTM 83. Springer-Verlag, New York, 1997. MR 97h:11130

[11] L. Yin, Stickelberger ideals and relative class numbers in function fields. J. Number Theory 81 (2000), no. 1, 162-169. MR 2001d:11114

Department of Mathematics Education, Chungbuk National University, Cheongue, Chungbuk, South Korea 361-763

E-mail address: hyjung@chungbuk.ac.kr

Department of Mathematics, Kaist, Daejon, South Korea 305-701

E-mail address: shbae@math.kaist.ac.kr

Department of Mathematics, Chungnam National University, Daejon, South Korea 305-764

E-mail address: jhahn@cnu.ac.kr 